\title{
Total electron ionization cross-sections for neutral molecules relevant to astrochemistry
}

\author{
David Heathcote and Claire Vallance* \\ Department of Chemistry, University of Oxford, Chemistry Research Laboratory, 12 \\ Mansfield Rd, Oxford OX1 3TA, UK \\ e-mail: claire.vallance@chem.ox.ac.uk
}

\begin{abstract}
The Binary-encounter Bethe model developed by Kim and Rudd $[1,2]$ has previously been used successfully to predict total electron ionisation cross sections for a wide variety of molecules. Here, we present total electron ionisation cross-sections calculated using the BEB model for a range of neutral molecules found in the interstellar medium, planetary and cometary atmospheres, and circumstellar shells. Many of these cross-sections are not amenable to experimental measurement due to the fact that the molecules of interest are highly chemically unstable under terrestrial conditions.
\end{abstract}

\section{Introduction}

To date, over 200 molecules have been detected in the interstellar medium or circumstellar shells $[3,4,5,6,7]$, with the list continuing to grow. The identified molecules, together with atomic species and other molecules postulated to be present, have been used in a variety of kinetic models of the astrochemical environments. Such models require specification of a network of reactions linking the various species, together with initial abundances of species present and rate constants for each reaction. Kinetic data for such models can be found in databases such as the Kinetic Database for Astrochemistry (KIDA) [8] and the UMIST Database for Astrochemistry [9]. Accurate modelling also requires knowledge of the physical conditions within the environment to be modelled, including temperature and density profiles, photon and cosmic ray fluxes, magnetic fields, and dust grain number densities and size ranges.

Electrons in interplanetary space originate from solar winds and from photoionization and Auger processes, and tend to have approximately MaxwellBoltzmann energy distributions spanning the range from several $\mathrm{eV}$ to several tens of $\mathrm{eV}$ [10]. A number of electron-induced atomic and molecular processes are relevant over this energy range. At the lowest energies, below the ionization threshold, elastic and inelastic scattering dominate over reactive or ionizing collisions, with elastic scattering continuing to be an important process up to energies of several hundred $\mathrm{eV}$. Dissociative electron attachment is also a key process at certain resonant energies below threshold. At higher energies, collision-induced dissociation, ionization, and dissociative ionization become important. The new Belgrade Electron Atom and Molecule Database (BEAMDB) [11], contains data for electron scattering processes in the form of differential and integrated cross sections, together with electron energy loss spectra.

Environments within which electron-induced processes are important are still being discovered, but it is clear that planetary and cometary atmospheres feature highly among them. To give a recent example, the Rosetta mission has offered a number of insights into the role of electrons in cometary comas [12]. The surprise 
observation of the O I line at $135.6 \mathrm{~nm}$ [13] in the far UV emission spectrum, coupled with the intensity ratio of the $\mathrm{H} \mathrm{I}$ and $\mathrm{O}$ I multiplets, and the observation of C I emissions, have been explained by electron dissociative excitation of $\mathrm{H}_{2} \mathrm{O}$ and $\mathrm{CO}_{2}$, respectively. Ion and electron sensors on the Rosetta craft have confirmed that there are copious amounts of electrons available to induce such processes.

In addition to modelling electron-induced processes in astrochemical environments, accurate electron ionization cross-sections are also required in order to allow quantitative analysis of mass spectrometric data from satellite missions. On-board mass spectrometers tend to employ electron ionization at $70 \mathrm{eV}$, close to the maximum in the ionization cross-section for many molecules.

Comprehensive modelling of electron ionization processes requires knowledge of both total and partial ionization cross-sections (defined respectively as the cross sections for ionizing the parent molecule and for forming specific fragment ions) as a function of collision energy, together with kinetic energy distributions of any fragment ions formed. Numerous methods have been developed to measure total and partial ionization cross sections [14]. Fragmentation patterns are available for many molecules that are sufficiently stable to be measured under terrestrial conditions (see for example [15]), but absolute cross-sections are difficult to measure and are not usually reported. Also, many molecules of interest to astrochemistry are not stable enough under terrestrial conditions to permit measurements to be made. Theoretical models therefore have an important role to play in providing cross-section data.

A number of theoretical models have been developed to determine total electron ionization cross-sections. Due to the complexity of modelling a system comprising two free electrons and an atomic or molecular ion in the exit channel, these methods are necessarily either semi-empirical or semi-classical for all but the simplest of systems. They are generally based on the additivity concept of Otvos and Stevenson [16], who postulated that the contribution of each molecular orbital to the total ionization cross-section can be calculated independently and the individual orbital cross-sections summed to give the total ionization cross-section of the molecule. A number of methods have been reviewed by Deutsch et al. [17] and by Harland and Vallance [14], and other methods have since been developed, such as Jain's complex spherical potential-ionization contribution (CSP-ic) method [18, 19, 20, 21, ?].

In the present work, we have chosen to employ the binary-encounter-Bethe (BEB) model developed by Kim and Rudd [1, 2], as it has been shown to reproduce experimentally-determined total electron ionization cross-sections with reasonable accuracy for a wide range of molecules $[2,22,23,24]$. We report total electron ionization cross-sections over the energy range from 0 to $5000 \mathrm{eV}$ for almost all neutral interstellar and circumstellar molecules listed in the Cologne Database for Molecular Spectroscopy $[3,4,5]$. We note that reliable theoretical methods for determining partial cross sections are yet to be developed, meaning that it is not yet possible to calculate all of the data required in order to analyse mass spectrometric data from unstable molecules, for which the fragmentation pattern is not known. While not standard practice on current satellite-borne instruments, this problem could be addressed by reducing the electron energy employed in the mass spectrometer's ion source to a value much closer to the ionization threshold for the species of interest. Near threshold, dissociative ionization is much reduced, and the ions produced are mostly parent ions, subject to the caveat that the lower ionization cross-section at these energies results in a considerable reduction in signal. 


\section{Methods}

\subsection{The binary-encounter-Bethe (BEB) model}

The BEB model is a simplified form of the binary-encounter-dipole (BED) model developed by the same authors $[1,2]$. The BED model combines two different theories describing the electron ionization process in a weighted combination. Modified Mott theory $[25,26]$ is used to describe small-impact-parameter or 'hard' collisions, and Bethe cross-section theory $[27,1,28]$ is used to describe large-impact-parameter or 'soft' collisions, which are dominated by dipole interactions. A switching function controls the relative weighting of the Mott and Bethe cross-sections at a given electron energy. The BEB model uses a simplified approach to calculating the differential dipole oscillator strength, a key parameter that appears in the BED model. The full derivation of the BED model and subsequent simplification to the BEB model is given in reference [1], with the resulting $\mathrm{BEB}$ cross section, $\sigma_{\mathrm{BEB}}$, given by

$$
\sigma_{\mathrm{BEB}}=\sum_{\text {orbitals }, i}\left\{\left(\frac{S_{i}}{t_{i}+\left(u_{i}+1\right)}\right)\left[\frac{\ln t_{i}}{2}\left(1-\frac{1}{t_{i}^{2}}\right)+\left(1-\frac{1}{t_{i}}-\frac{\ln t_{i}}{t_{i}+1}\right)\right]\right\}(1)
$$

with

$$
t_{i}=\frac{T}{B_{i}} \quad u_{i}=\frac{U_{i}}{B_{i}} \quad S_{i}=4 \pi a_{0}^{2} N_{i}\left(\frac{R_{\infty}}{B_{i}}\right)^{2}
$$

In the above, the sum is over the atomic or molecular orbitals of the atom or molecule. $T$ is the kinetic energy of the incident electron, $B_{i}$ and $U_{i}$ are the orbital binding energy and average kinetic energy of the ejected electron, respectively, and $N_{i}$ is the orbital occupation number. The parameters $a_{0}$ and $R_{\infty}$ are the Bohr radius and the Rydberg constant, respectively. It should be noted that within the calculation, $T$ must be greater than $B_{i}$ for a given molecular orbital in order to generate a non-zero cross-section, i.e. the incident electron must have sufficient energy to overcome the binding energy of the bound electron. From a computational point of view, determining the contribution to the total ionization cross section from a given molecular orbital requires only the calculation of the binding energy and kinetic energy of the electrons within the orbital. Summing over the orbitals yields the total electron ionization cross-section. The accuracy of the calculated orbital binding and kinetic energies is reflected directly in the accuracy of the resulting BEB cross-section, with the parameters for the highest-lying orbitals having the greatest effect [2]. The computational methods used to determine these parameters are discussed in Section 2.2 .

The BEB model has been shown to reproduce the total electron ionization cross section satisfactorily for a wide range of molecules [2, 22, 23, 24], and forms the basis for the electron impact ionization cross-section database hosted by NIST [29]. A

relativistic correction has also been developed [28], but is only required for incident electron energies above $20 \mathrm{keV}$, and is therefore not employed in the present work, which spans the range up to $5 \mathrm{keV}$.

\subsection{Computational methods}

As noted above, high-quality orbital kinetic energies and binding energies are required as input to the BEB model in order to obtain reliable results. We have previously [23] performed a comprehensive benchmarking study to determine the 


\begin{aligned} & \hline Molecule Computational method \\ & \hline $\mathrm{C}_{4} \mathrm{H}$ MP2/cc-pVTZ; MP2/aug-cc-pVTZ; UHF/aug-cc-pVTZ \\ & $\mathrm{C}_{5} \mathrm{H}$ ROHF/aug-cc-pVTZ; MP2/aug-cc-pVTZ \\ & $\mathrm{C}_{5} \mathrm{~N}$ MP2/cc-pVTZ; MP2/aug-cc-pVTZ; UHF/aug-cc-pVTZ \\ & $\mathrm{C}_{6} \mathrm{H}$ MP2/aug-cc-pVTZ \\ & \hline\end{aligned}

Table 1. Non-standard methods used for geometry optimisation in the case of $\mathrm{C}_{4} \mathrm{H}, \mathrm{C}_{5} \mathrm{H}, \mathrm{C}_{5} \mathrm{~N}$, and $\mathrm{C}_{6} \mathrm{H}$ in order to achieve convergence at the same level of theory as used for all other molecules.

optimal computational methodology to employ when determining these parameters, and we employ these optimised electronic structure methods in the present study. Our approach, outlined below, offers a good balance between the accuracy of the molecular parameters and computational time. All calculations were performed using the Gaussian 09 electronic structure package [30].

Molecular geometries were optimised at HF/aug-cc-pVTZ level [31, 32, 33]. Restricted (RHF) and unrestricted (UHF) Hartree-Fock theories were used for closedshell and open-shell molecules, respectively [34, 35, 36]. Following the geometry optimisation, orbital binding energies were calculated using partial third-order (P3) electron propagator theory [37]. This approach has been shown previously [23] to account sufficiently well for electron correlation that accurate ionization potentials and binding energies are obtained. A plot of the P3 binding energies vs the HF energies yields a linear scaling factor that accounts for the effect of electron correlation on the binding energies. The orbital kinetic energies were scaled by the same factor in order to ensure that the virial theorem is satisfied.

A Python 2.7 script was written to automate both the electronic structure calculations, given an initial-guess geometry as input, and the subsequent calculation of the $\mathrm{BEB}$ electron ionization cross section, $\sigma_{\mathrm{BEB}}$, using Equation 1.

For a few of the molecules studied, it was not possible to employ the method described above, and the most accurate electronic structure methods for which converged results could be obtained were used instead. Orbital parameters for the single molecular orbital of the $\mathrm{H}_{2}$ molecule were calculated at the $\operatorname{CCSD}(\mathrm{T}) /$ augcc-pVTZ level of theory. For $\mathrm{C}_{4} \mathrm{H}, \mathrm{C}_{5} \mathrm{H}, \mathrm{C}_{6} \mathrm{H}, \mathrm{C}_{7} \mathrm{H}$, and $\mathrm{C}_{5} \mathrm{~N}$, the calculations as described above did not converge in the first instance. However, by using either a different self-consistent field (SCF) method, or by initially removing the diffuse orbitals from the basis set (i.e. using the cc-pVTZ basis set) for the geometry optimisation, an initial geometry could be found for which convergence was achieved in the P3 accurate energy calculation. $\mathrm{C}_{7} \mathrm{H}$ required an initial geometry calculation at the MP2 level of theory, in which the direct inversion in the iterative subspace method [38] was turned off. From this geometry, optimised geometries were obtained for both MP2 and UHF levels of theory, with the cross-section determined from the UHF geometry optimisation reported here for consistency with the calculations on other molecules. The geometry optimisation methods used for the other four molecules are listed in Table 1.

\section{Results}

Figure 1 shows the calculated total electron ionization cross-sections for a selection of the molecules studied over the energy range from 0 to $5000 \mathrm{eV}$. Table 2 shows the 

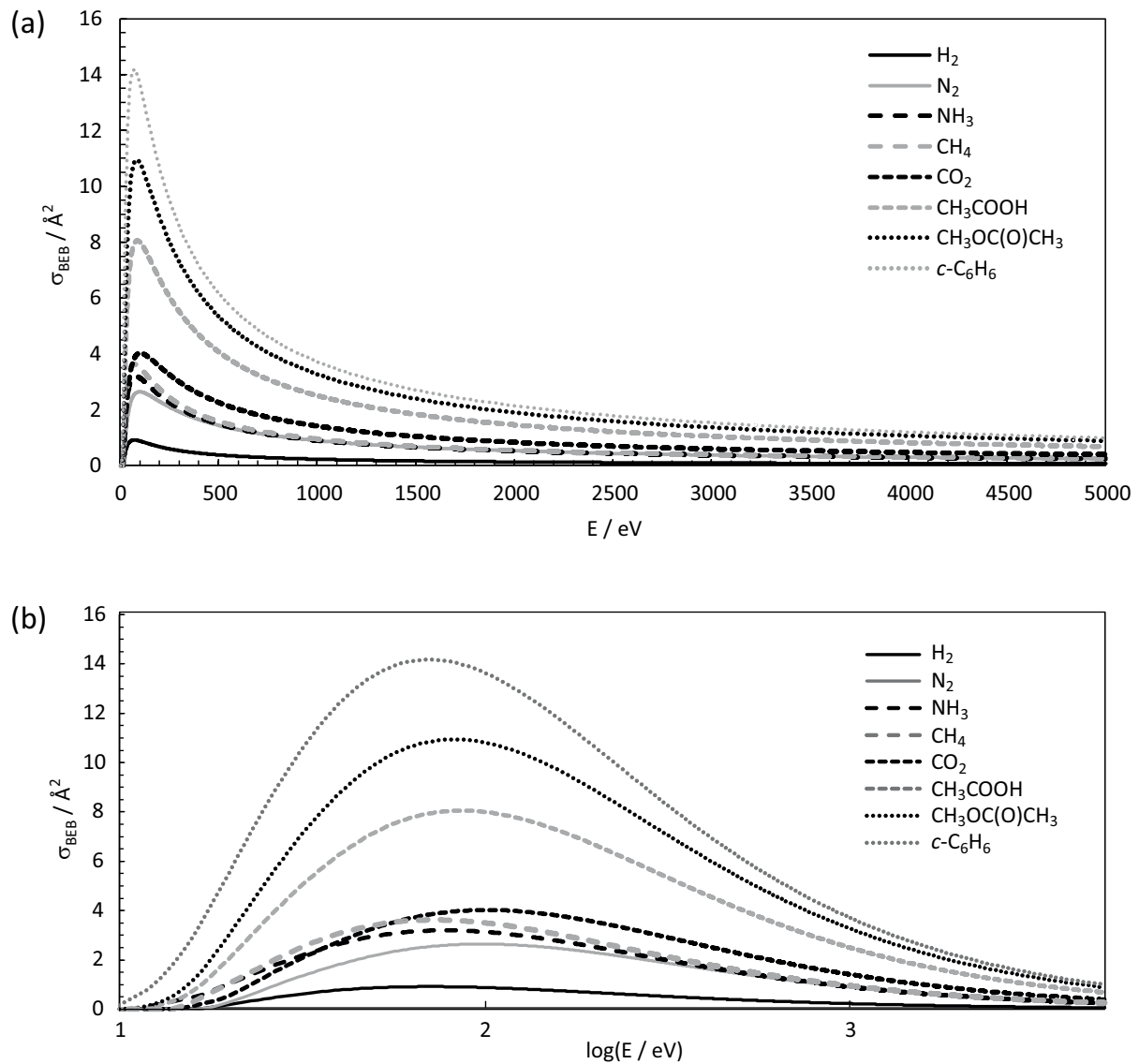

Figure 1. Electron ionization cross-sections calculated using the BEB model for a selection of molecules. Cross sections are plotted from $0-5,000 \mathrm{eV}$ on (a) a linear and (b) a log scale.

maximum cross-sections and corresponding electron energies for all neutral molecules studied. Plots of the cross-sections for the molecules listed in Table 2 over the electron energy range from 0 to $300 \mathrm{eV}$ are shown in Figures 2 and 3, and the complete data set for all molecules over the range $0-5000 \mathrm{eV}$ is available in the Supplementary Information.

Comparison with experimental data shows that in general, the BEB model tends to overestimate the ionization cross-section at low electron energies, whilst underestimating the cross-section at higher electron energies. The overestimation at low energies can be attributed to the fact that the BEB model assumes that any interaction between the electron and molecule results in the formation of an ion. However, this is not necessarily the case, as molecular excitation or neutral dissociation processes are also possible outcomes of the electron-molecule collision. At higher energies, the underestimation can be attributed to the occurrence of multiple ionization processes; the $\mathrm{BEB}$ method assumes that each electron-molecule collision 


\begin{tabular}{|c|c|c|c|c|c|}
\hline Molecule & $\sigma_{\max } / \check{A}^{2}$ & $\mathrm{EE}_{\max } / \mathrm{eV}$ & Molecule & $\sigma_{\max } / \stackrel{\AA}{A}^{2}$ & $\mathrm{EE}_{\max } / \mathrm{eV}$ \\
\hline $\mathrm{H}_{2}$ & 0.92 & 71 & $\mathrm{CH}_{3} \mathrm{CHO}$ & 6.72 & 82 \\
\hline $\mathrm{HF}$ & 1.72 & 113 & $\mathrm{H}_{2} \mathrm{CCNH}$ & 6.73 & 75 \\
\hline $\mathrm{OH}$ & 2.03 & 95 & $\mathrm{HC}_{3} \mathrm{~N}$ & 6.76 & 80 \\
\hline $\mathrm{NH}$ & 2.25 & 83 & $\mathrm{HC}_{2} \mathrm{NC}$ & 6.97 & 78 \\
\hline $\mathrm{H}_{2} \mathrm{O}$ & 2.46 & 92 & $\mathrm{H}_{2} \mathrm{CCHOH}$ & 7.03 & 79 \\
\hline $\mathrm{N}_{2}$ & 2.64 & 98 & $\mathrm{HNCHCN}$ & 7.24 & 82 \\
\hline $\mathrm{CO}$ & 2.72 & 96 & $\mathrm{HC}_{2} \mathrm{CHO}$ & 7.49 & 81 \\
\hline $\mathrm{CH}$ & 2.74 & 67 & $\mathrm{HNC}_{3}$ & 7.54 & 73 \\
\hline $\mathrm{NH}_{2}$ & 2.76 & 80 & $\mathrm{c}-\mathrm{H}_{2} \mathrm{C} 3 \mathrm{O}$ & 7.68 & 81 \\
\hline $\mathrm{NO}$ & 2.98 & 97 & $\mathrm{CH}_{3} \mathrm{NCO}$ & 7.69 & 84 \\
\hline $\mathrm{O}_{2}$ & 2.98 & 103 & $\mathrm{C}_{4} \mathrm{H}$ & 7.71 & 71 \\
\hline $\mathrm{CH}_{2}$ & 3.12 & 67 & $\mathrm{CH}_{3} \mathrm{CHNH}$ & 7.74 & 75 \\
\hline $\mathrm{CN}$ & 3.16 & 84 & $\mathrm{CH}_{3} \mathrm{C}_{2} \mathrm{H}$ & 7.81 & 68 \\
\hline $\mathrm{NH}_{3}$ & 3.2 & 78 & $\mathrm{CH}_{2} \mathrm{CHCN}$ & 7.88 & 78 \\
\hline $\mathrm{C}_{2}$ & 3.27 & 78 & $\mathrm{CH}_{3} \mathrm{COOH}$ & 8.05 & 87 \\
\hline $\mathrm{CH}_{3}$ & 3.38 & 70 & $\left(\mathrm{CH}_{3}\right)_{2} \mathrm{O}$ & 8.05 & 78 \\
\hline $\mathrm{HCN}$ & 3.5 & 84 & $\mathrm{CH}_{3} \mathrm{CH}_{2} \mathrm{OH}$ & 8.07 & 78 \\
\hline $\mathrm{HNO}$ & 3.61 & 92 & $\mathrm{l}-\mathrm{HC}_{4} \mathrm{H}$ & 8.08 & 72 \\
\hline $\mathrm{CH}_{4}$ & 3.62 & 73 & $\mathrm{HC}(\mathrm{O}) \mathrm{OCH}_{3}$ & 8.15 & 86 \\
\hline $\mathrm{HO}_{2}$ & 3.63 & 97 & $\mathrm{CH}_{2} \mathrm{OHCHO}$ & 8.18 & 86 \\
\hline $\mathrm{HCO}$ & 3.66 & 86 & $\mathrm{c}-\mathrm{C}_{2} \mathrm{H}_{4} \mathrm{O}$ & 8.22 & 71 \\
\hline $\mathrm{HNC}$ & 3.7 & 81 & $\mathrm{C}_{4} \mathrm{H}_{2}$ & 8.23 & 71 \\
\hline $\mathrm{CO}_{2}$ & 4.02 & 102 & $\mathrm{C}_{3} \mathrm{H}_{6}$ & 8.31 & 71 \\
\hline $\mathrm{H}_{2} \mathrm{CO}$ & 4.1 & 86 & $\mathrm{H}_{2} \mathrm{NCH}_{2} \mathrm{CN}$ & 8.33 & 80 \\
\hline $\mathrm{N}_{2} \mathrm{O}$ & 4.11 & 99 & $\mathrm{CH}_{2} \mathrm{CHCHO}$ & 8.51 & 80 \\
\hline $\mathrm{C}_{2} \mathrm{H}$ & 4.12 & 72 & $\mathrm{C}_{5}$ & 8.57 & 73 \\
\hline $\mathrm{H}_{2} \mathrm{O}_{2}$ & 4.23 & 93 & $\mathrm{CH}_{3} \mathrm{CH}_{2} \mathrm{CN}$ & 8.75 & 78 \\
\hline $\mathrm{H}_{2} \mathrm{CN}$ & 4.39 & 79 & $\mathrm{CH}_{3} \mathrm{C}(\mathrm{O}) \mathrm{NH}_{2}$ & 9.16 & 81 \\
\hline $\mathrm{C}_{2} \mathrm{H}_{2}$ & 4.52 & 73 & $\mathrm{l}-\mathrm{HC}_{4} \mathrm{~N}$ & 9.2 & 75 \\
\hline $\mathrm{CH}_{3} \mathrm{O}$ & 4.74 & 83 & $\mathrm{CH}_{3} \mathrm{NHCHO}$ & 9.24 & 80 \\
\hline $\mathrm{C}_{2} \mathrm{O}$ & 4.82 & 83 & $\mathrm{CH}_{3} \mathrm{C}_{3} \mathrm{~N}$ & 9.41 & 78 \\
\hline $\mathrm{HNCO}$ & 4.93 & 90 & $\left(\mathrm{CH}_{3}\right)_{2} \mathrm{CO}$ & 9.46 & 79 \\
\hline $\mathrm{HOCN}$ & 4.94 & 90 & $\mathrm{C}_{5} \mathrm{H}$ & 9.52 & 71 \\
\hline $\mathrm{H}_{2} \mathrm{CNH}$ & 4.96 & 77 & $\mathrm{CH}_{3} \mathrm{CH}_{2} \mathrm{CHO}$ & 9.61 & 79 \\
\hline $\mathrm{C}_{2} \mathrm{~N}$ & 4.99 & 80 & $\mathrm{C}_{5} \mathrm{~N}$ & 9.67 & 78 \\
\hline $\mathrm{C}_{2} \mathrm{H}_{4}$ & 5.07 & 72 & $\mathrm{R}-\mathrm{CH}_{3} \mathrm{CHCH}_{2} \mathrm{O}$ & 9.71 & 78 \\
\hline $\mathrm{HCNO}$ & 5.14 & 88 & $\mathrm{~S}-\mathrm{CH}_{3} \mathrm{CHCH}_{2} \mathrm{O}$ & 9.71 & 78 \\
\hline $\mathrm{C}_{3}$ & 5.25 & 75 & $\mathrm{CH}_{2} \mathrm{CCHCN}$ & 9.72 & 77 \\
\hline $\mathrm{CH}_{3} \mathrm{OH}$ & 5.26 & 82 & $\left(\mathrm{CH}_{2} \mathrm{OH}\right)_{2}$ & 9.77 & 82 \\
\hline $\mathrm{HCCO}$ & 5.34 & 82 & $\mathrm{HC}_{5} \mathrm{~N}$ & 10.16 & 78 \\
\hline $\mathrm{HCOOH}$ & 5.41 & 93 & $\mathrm{C}_{2} \mathrm{H}_{5} \mathrm{OCHO}$ & 10.86 & 83 \\
\hline $\mathrm{HCCN}$ & 5.6 & 78 & $\mathrm{CH}_{3} \mathrm{OC}(\mathrm{O}) \mathrm{CH}_{3}$ & 10.94 & 83 \\
\hline $\mathrm{H}_{2} \mathrm{C}_{2} \mathrm{O}$ & 5.72 & 82 & $\mathrm{C}_{2} \mathrm{H}_{5} \mathrm{OCH}_{3}$ & 10.98 & 76 \\
\hline $\mathrm{c}-\mathrm{C}_{3} \mathrm{H}$ & 5.82 & 73 & $\mathrm{C}_{6} \mathrm{H}$ & 11.06 & 72 \\
\hline $\mathrm{H}_{2} \mathrm{CCN}$ & 5.87 & 79 & $\mathrm{i}-\mathrm{C}_{3} \mathrm{H}_{7} \mathrm{CN}$ & 11.41 & 77 \\
\hline $\mathrm{CH}_{3} \mathrm{CN}$ & 6.05 & 80 & $\mathrm{n}-\mathrm{C}_{3} \mathrm{H}_{7} \mathrm{CN}$ & 11.53 & 77 \\
\hline $\mathrm{H}_{2} \mathrm{NCN}$ & 6.09 & 81 & $\mathrm{CH}_{3} \mathrm{C}_{4} \mathrm{H}$ & 11.54 & 69 \\
\hline $\mathrm{CH}_{3} \mathrm{NH}_{2}$ & 6.09 & 75 & $1-\mathrm{HC}_{6} \mathrm{H}$ & 11.64 & 72 \\
\hline $1-\mathrm{C}_{3} \mathrm{H}$ & 6.18 & 70 & $\mathrm{C}_{6} \mathrm{H}_{2}$ & 11.89 & 70 \\
\hline $\mathrm{HNCNH}$ & 6.18 & 79 & $\mathrm{CH}_{3} \mathrm{C}_{5} \mathrm{~N}$ & 12.99 & 76 \\
\hline $\mathrm{CH}_{3} \mathrm{NC}$ & 6.25 & 78 & $\mathrm{C}_{7} \mathrm{H}$ & 13.54 & 69 \\
\hline $\mathrm{HC}(\mathrm{O}) \mathrm{CN}$ & 6.34 & 89 & $\mathrm{HC}_{7} \mathrm{~N}$ & 13.61 & 77 \\
\hline $\mathrm{C}_{3} \mathrm{O}$ & 6.35 & 81 & $\mathrm{c}-\mathrm{C}_{6} \mathrm{H}_{6}$ & 14.18 & 71 \\
\hline $\mathrm{NH}_{2} \mathrm{CHO}$ & 6.41 & 84 & $\mathrm{CH}_{3} \mathrm{C}_{6} \mathrm{H}$ & 15.01 & 69 \\
\hline $\mathrm{c}-\mathrm{C}_{3} \mathrm{H}_{2}$ & 6.47 & 72 & $\mathrm{C}_{8} \mathrm{H}$ & 15.14 & 70 \\
\hline $1-\mathrm{C}_{3} \mathrm{H}_{2}$ & 6.55 & 71 & $\mathrm{HC}_{9} \mathrm{~N}$ & 17.09 & 76 \\
\hline $\mathrm{C}_{3} \mathrm{~N}$ & 6.66 & 79 & $\mathrm{HC}_{11} \mathrm{~N}$ & 20.35 & 75 \\
\hline
\end{tabular}

Table 2. The maxima in the electron ionization cross-sections, $\sigma_{\max }$, and associated electron energies, $\mathrm{EE}_{\max }$, determined for neutral molecules containing only light atoms. 

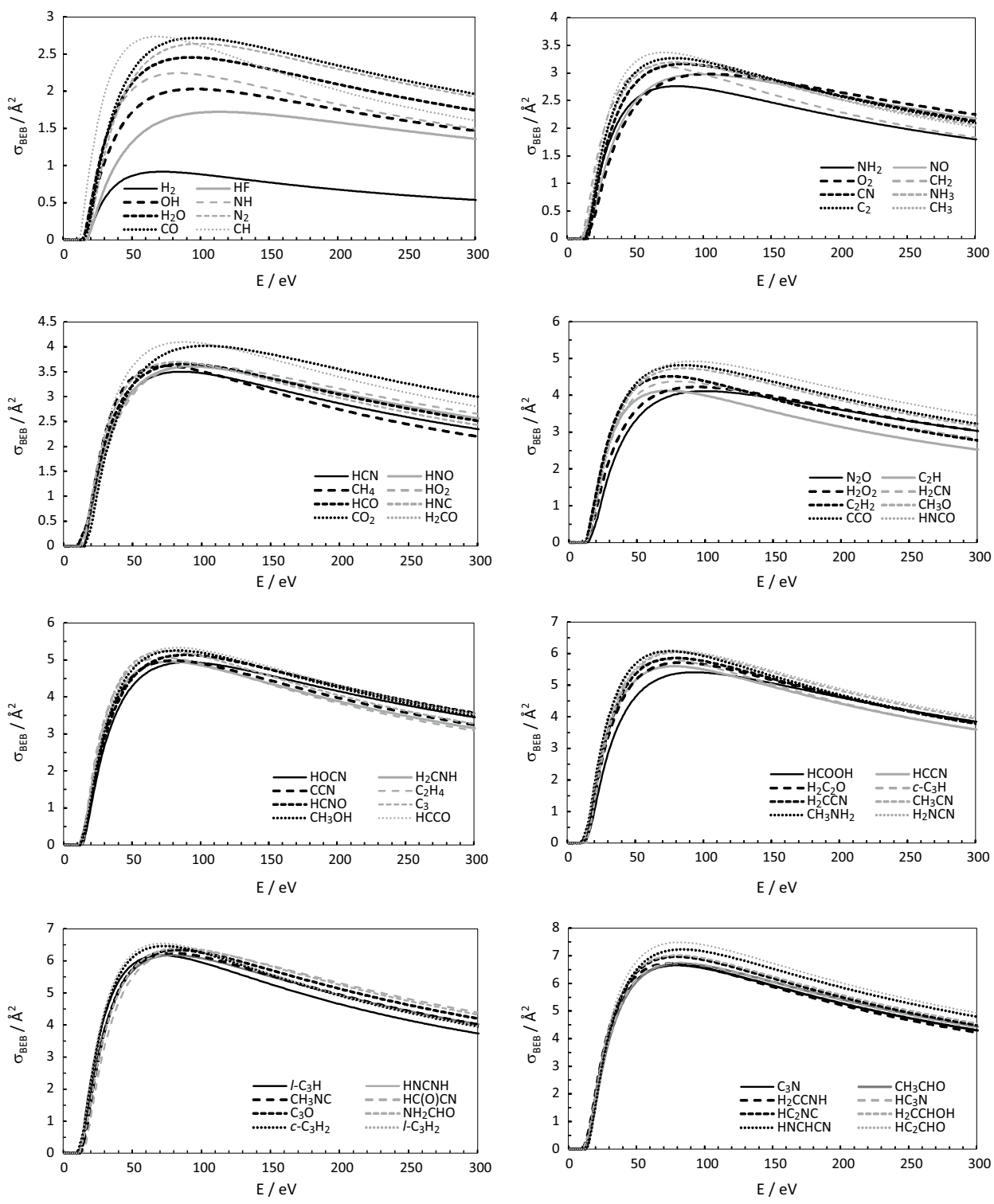

Figure 2. Electron ionization cross-sections calculated using the BEB model over the electron energy range from 0 to $300 \mathrm{eV}$ for molecules containing first and second row atoms. Cross-sections are plotted in order of increasing maximum cross-section

yields a singly charged ion, whereas double and even triple ionization can occur at sufficiently high electron energies. In an experiment, the cross-section is determined from a measurement of the ion current, and the presence of doubly or triply charged ions yields an increased current, and therefore an increase in the measured crosssection, relative to that resulting from singly charged ions only. 

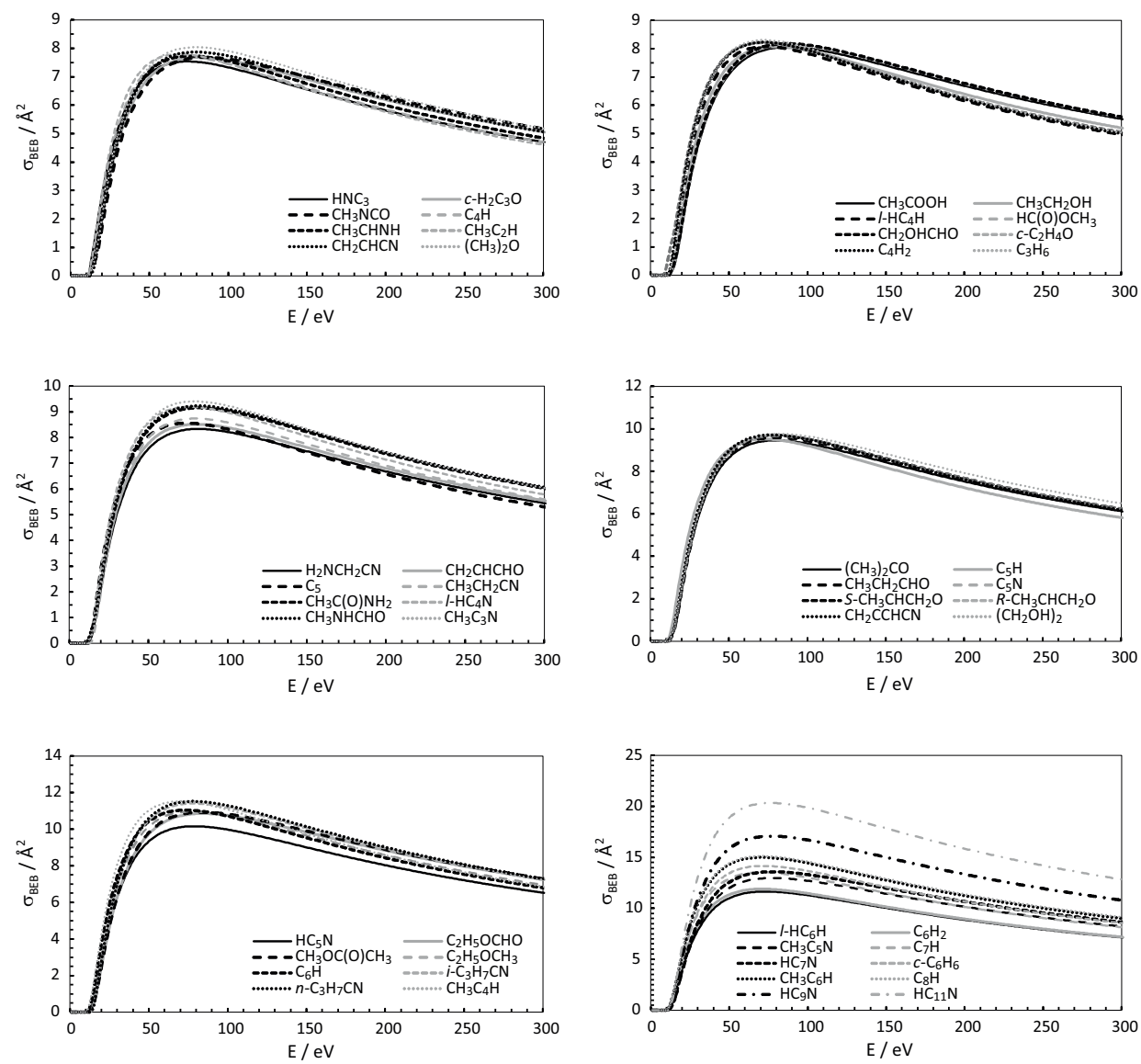

Figure 3. Continuation of Figure 2.

\subsection{Molecules containing heavy atoms}

Numerous molecules have been observed in interstellar and circumstellar media that contain atoms from the third row of the periodic table or below. It is well known that the BEB model as presented in Section 2.1 significantly underestimates the electron ionization cross-section relative to experimental measurements, and a number of different corrections to the orbital kinetic energy have been proposed in order to address this discrepancy $[2,39,40]$. These attempt to account for the fact that the electron kinetic energy is much greater in regions near the core than in the outer regions of the orbital, but that ionization is much more likely to occur outside the core. When considering electron ionization, the 'effective' kinetic energy is therefore lower than the average orbital kinetic energy returned from the electronic structure calculations. One of the most problematic aspects of this approach lies in identifying the appropriate threshold for the contribution of 'heavy atom' atomic orbitals to each molecular orbital at which to trigger the kinetic energy correction. We have explored several of the proposed corrections in calculating electron ionization cross-sections for molecules containing heavy atoms, unfortunately with fairly unconvincing results. 
An alternative approach, proposed by Huo and Kim [39], employs relativistic effective core potentials. These significantly reduce the penetration of the valence electrons into the core, achieving a reduction in orbital kinetic energy that increases with atomic number. This method removes the reliance on defining a somewhat arbitrary threshold atomic orbital contribution, reduces the computational cost for the core electrons, and includes scalar relativistic effects [40].

It is clear that further work is needed in order to apply the BEB model reliably to molecules containing heavy atoms, and rather than present what we believe to be unreliable data here, we plan to evaluate both correction methods in a more comprehensive future study once a greater body of experimental data is available for comparison.

\subsection{Parameterised cross-sections}

For the purposes of employing our calculated cross-sections in computational models, we have fitted each energy dependent cross-section to the following analytical function, which is widely used in simulations of fusion edge plasmas [41]:

$$
\sigma(E)=\left(\frac{a_{1}}{E}\right)\left[1-\frac{E_{t}}{E}\right]^{a_{2}}\left[\ln \left(\frac{E}{E_{t}}\right)+a_{3}+a_{4}\left(\frac{E_{t}}{E}\right)\right]
$$

Here, $E$ is the incident electron energy and $E_{t}$ is the first ionization energy (ionization threshold), both in units of $\mathrm{eV}$. In the fits reported here, the $E_{t}$ values were fixed at the values determined from the $a b$ initio calculations described in Section 2.2. The cross-section, $\sigma(E)$ is expressed in units of $\AA^{2}$, the first fitting parameter, $a_{1}$, has units of $\AA^{2} \mathrm{eV}$, and the remaining fitting parameters, $a_{2}$ to $a_{4}$, are dimensionless. The parameters $E_{t}, a_{1}, a_{2}, a_{3}$, and $a_{4}$ for each fitted cross section are provided in the Supplementary Information.

To demonstrate the performance of the fitting function, Figures 4(a) and (b) show the calculated BEB cross-sections and fits for the molecules $\mathrm{N}_{2}$ and $\mathrm{CH}_{4}$, respectively, over the energy range from 0 to $300 \mathrm{eV}$. The two functions plotted over the full energy range from 0 to $5000 \mathrm{eV}$ are shown as insets to each figure. Experimentally measured cross-sections $[42,43,44,45,46,47]$ from a few different research groups are also shown, together with previous BEB calculations [2] employing a different protocol from the present work for the electronic structure calculations. The fitting function is able to match the BEB cross-section to better than $5 \%$ over the full energy range. However, when looking at the $0-300 \mathrm{eV}$ plots we see that up to an energy of about $50 \mathrm{eV}$ the fitting function tends to underestimate the BEB cross section slightly, while the cross-section is overestimated near the peak of the ionization efficiency curve. For $\mathrm{N}_{2}$, the predicted cross-sections are in good agreement both with the experimental data and with the previously-reported $\mathrm{BEB}$ calculation. For $\mathrm{CH}_{4}$, the $\mathrm{BEB}$ model agrees well with the measured cross-sections below about $40 \mathrm{eV}$, but significantly underestimates the cross-section at high energies. This may be due to the fact that the BEB model does not include the effects of double ionization. Most experiments record the ion current to determine the ionization cross-section, with the consequence that doubly charged ions contribute twice as much to the signal as singly charged ions. The resulting cross section is known as a gross (as opposed to counting) ionization cross-section, i.e.

$$
\sigma=\sigma_{1}+2 \sigma_{2}+3 \sigma_{3}+\ldots
$$


where $\sigma$ is the measured cross-section, and $\sigma_{1}, \sigma_{2}$, and $\sigma_{3}$ are the cross-sections for single, double, and triple ionization, respectively. The double ionization threshold for $\mathrm{CH}_{4}$ is $38 \mathrm{eV}$, so we might expect doubly charged ions to start contributing to the total cross-section for energies higher than this value, possibly explaining the observed discrepancy between experiment and theory. The double ionization threshold for $\mathrm{N}_{2}$ is only a few $\mathrm{eV}$ higher than for $\mathrm{CH}_{4}$, so we might expect a similar contribution to the signal from doubly charged ions. However, for $\mathrm{N}_{2}$ some of the experimental data sets predict cross-sections lower than the BEB predictions, and some higher, so it is difficult to draw any firm conclusions within the experimental uncertainties.
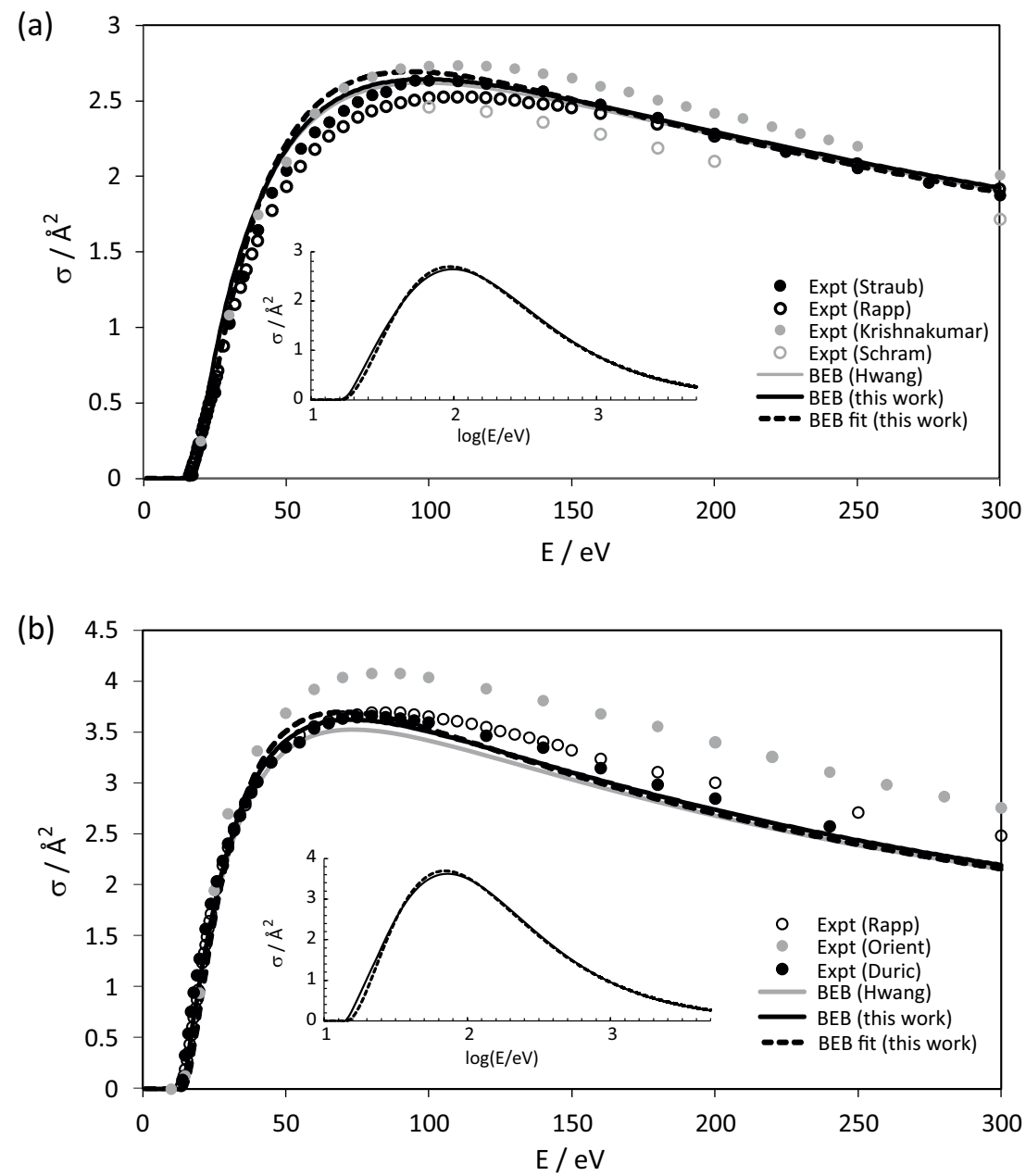

Figure 4. BEB cross-sections and fits to Equation (3) for (a) $\mathrm{N}_{2}$ and (b) $\mathrm{CH}_{4}$, together with previously published BEB calculations[2] and experimental data[42, 43, 44, 45, 46, 47] for comparison. BEB cross-sections and fits from this work over the entire energy range from $0-5000 \mathrm{eV}$ are shown in the insets. 


\section{Conclusions}

We have reported total electron ionisation cross-sections calculated using the BEB model for the majority of neutral molecules reported in the interstellar medium and circumstellar shells. Most of these molecules are not amenable to experimental measurement due to their chemical instability under the relatively high pressure and high temperature conditions of a terrestrial laboratory. Further work is required in order to optimise the BEB model for molecules containing heavy atoms, and we plan to investigate these aspects of the model in greater depth when additional experimental data becomes available for comparison. The calculated cross-sections have been fitted to a widely used parameterised fitting function for ease of use in modelling applications.

\section{Supplementary information}

Tabulated cross sections over the energy range from 0 to $5000 \mathrm{eV}$ for all of the molecules studied are available in the Supplementary Information as an Excel file. Fitting parameters obtained from non-linear fits of Equation (3) to each data set are also provided.

\section{Acknowledgements}

The authors would like to thank Weiwei Zhou for preparing the data presented in Figure 4, and to acknowledge support from the EPSRC via Programme Grant EP/L005913/1 and a Doctoral Training Grant studentship to DH.

\section{References}

[1] Kim Y K and Rudd M E 1994 Phys. Rev. A 50 3954-3967

[2] Hwang W, Kim Y and Rudd M E 1996 J. Chem. Phys. 104 2956-2966

[3] Müller H S P, Thorwirth S, Roth D A and Winnewisser G 2001 Astron. Astrophys. 370 L49-L52

[4] Müller H S P, Schlöder F, Stutzki J and Winnewisser G 2005 J. Mol. Struct. 742 215-227 ISSN $0022-2860$

[5] Müller H S P, Endres C P, Stutzki J and Schlemmer S 2001 Molecules in space http://www.astro.uni-koeln.de/cdms/molecules online: accessed 22 November 2016

[6] Herbst E and Jr J T Y 2013 Chem. Rev. 113(12) 8707 - 8709

[7] Herbst E 2017 Int. Rev. Phys. Chem. 36(2) $287-331$

[8] Wakelam V, Herbst E, Loison J C, Smith I W M, Chandrasekaran V, Pavone B, Adams N G, Bacchus-Montabonel M C, Bergeat A, Béroff K, Bierbaum V M, Chabot M, Dalgarno A, van Dishoeck E F, Faure A, Geppert W D, Gerlich D, Galli D, Hébrard E, Hersant F, Hickson K M, Honvault P, Klippenstein S J, Picard S L, Nyman G, Pernot P, Schlemmer S, Selsis F, Sims I R, Talbi D, Tennyson J, Troe J, Wester R and Wiesenfeld L 2012 The Astrophysical Journal Supplement Series 19921

[9] McElroy D, Walsh C, Markwick A J, Cordiner M A, Smith K and Millar T J 2013 Astron. Astrophys. 550 A36

[10] Burch J L, Goldstein R, Cravens T E, Gibson W C, Lundin R N, Pollock C J, Winningham J D and Young D T 2007 Space Sci. Rev. 128 697-712

[11] Marinković B P, Jevremović D, Srećković V A, Vujčić V, Ignjatović L M, Dimitrijević M S and Mason N J 2017 Eur. Phys. J. D $\mathbf{7 1}$

[12] Marinković B P, Bredehöft J H, Vujčić V, Jevremović D and Mason N J 2017 Atoms 546

[13] Feldman P D, A'Hearn M F, Bertaux J L, Feaga L M, Parker J W, Schindhelm E, Steffl A J, Stern S A, Weaver H A and et al H S 2015 Astron. Astrophys. 583 A8

[14] Harland P W and Vallance C 1998 Advances in Gas Phase Ion Chemistry (Elsevier Science) ISBN 9780080550862

[15] Nist chemistry webbook https://webbook.nist.gov

[16] Otvos J W and Stevenson D P 1956 J. Am. Chem. Soc. 78 546-551 ISSN 0002-7863 
[17] Deutsch H, Becker K, Matt S and Märk T D 2000 Int. J. Mass spectrom. 197 37-69 ISSN $1387-3806$

[18] Jain A and Baluja K L 1992 Phys. Rev. A 45202

[19] Joshipura K N, Vinodkumar M and Patel M U 2001 J. Phys. B 34509 ISSN 0953-4075

[20] Joshipura K N, Vinodkumar M, Limbachiya C G and Antony B K 2004 Phys. Rev. A 69(2) 022705

[21] Limbachiya C, Vinodkumar M and Mason N 2011 Phys. Rev. A 83042708

[22] Kim Y K, Hwang W, Weinberger N M, Ali M A and Rudd M E 1997 J. Chem. Phys. 106 $1026-1033$

[23] Bull J N, Harland P W and Vallance C 2012 J. Phys. Chem. A 116 767-777 ISSN 1089-5639

[24] Bull J N, Lee J W L and Vallance C 2014 Phys. Chem. Chem. Phys. 16 10743-10752 ISSN $1463-9076$

[25] Mott N F 1930 Proc. R. Soc. London, Ser. A 126 259-267

[26] Vriens L 1969 Case studies in atomic collision physics vol 1 (North-Holland Publishing Company)

[27] Bethe H 1930 Ann. Phys. (Leipzig) 397 325-400 ISSN 1521-3889

[28] Kim Y K, Santos J P and Parente F 2000 Phys. Rev. A 62052710 pRA

[29] Kim Y K, Irikura K K, Rudd M E, Ali M A, Stone P M, Chang J, Coursey J S, Dragoset R A, Kishore A R, Olsen K J, Sansonetti A M, Wiersma G G, Zucker D S and Zucker M A 1997 Electron-impact cross sections for ionization and excitation database https://www.nist.gov/pml/electron-impact-cross-sections-ionization-and-excitation-database

[30] Frisch M J, Trucks G W, Schlegel H B, Scuseria G E, Robb M A, Cheeseman J R, Scalmani G, Barone V, Mennucci B, Petersson G A, Nakatsuji H, Caricato M, Li X, Hratchian H P, Izmaylov A F, Bloino J, Zheng G, Sonnenberg J L, Hada M, Ehara M, Toyota K, Fukuda R, Hasegawa J, Ishida M, Nakajima T, Honda Y, Kitao O, Nakai H, Vreven T, Jr J A M, Peralta J E, Ogliaro F, Bearpark M, Heyd J J, Brothers E, Kudin K N, Staroverov V N, Kobayashi R, Normand J, Raghavachari K, Rendell A, Burant J C, Iyengar S S, Tomasi J, Cossi M, Rega N, Millam J M, Klene M, Knox J E, Cross J B, Bakken V, Adamo C, Jaramillo J, Gomperts R, Stratmann R E, O Yazyev A J A, Cammi R, Pomelli C, Ochterski J W, Martin R L, Morokuma K, Zakrzewski V G, Voth G A, Salvador P, Dannenberg J J, Dapprich S, Daniels A D, Farkas O, Foresman J B, Ortiz J V, Cioslowski J and Fox D J 2009 Gaussian 09 Gaussian, Inc., Wallingford CT

[31] Dunning T H 1989 J. Chem. Phys. 90 1007-1023

[32] Kendall R A, Dunning T H and Harrison R J 1992 J. Chem. Phys. 96 6796-6806

[33] Woon D E and Dunning T H 1993 J. Chem. Phys. 98 1358-1371

[34] Roothaan C C J 1951 Reviews of Modern Physics 23 69-89 rMP

[35] Pople J A and Nesbet R K 1954 J. Chem. Phys. 22 571-572 uHF

[36] McWeeny R and Diercksen G 1968 J. Chem. Phys. 49 4852-4856

[37] Ortiz J V 1996 J. Chem. Phys. 104 7599-7605

[38] Pulay P 1982 J. Comput. Chem. 3 556-560 ISSN 1096-987X

[39] Huo W M and Kim Y K 2000 Chem. Phys. Lett. 319 576-586 ISSN 0009-2614

[40] Scott G E and Irikura K K 2005 J. Chem. Theory Comput. 1 1153-1161 ISSN 1549-9618

[41] Huber S E, Mauracher A, Sukuba I, Urban J, Maihom T and Probst M 2017 Eur. Phys. J. D 71335

[42] Straub H C, Renault P, Lindsay B G, Smith K A and Stebbings R F 1996 Phys. Rev. A 542146

[43] Rapp D and Englander-Golden P 1965 J. Chem. Phys. 431464

[44] Krishnakumar E and Srivastava S K 1990 J. Phys. B 231893

[45] Schram B L, Moustafa H R, Schutten J and de Heer F J 1966 Physica 32734

[46] Orient O J and Srivastava S K 1987 J. Phys. B 203923

[47] Durić N, Čadež I and Kurepa M 1991 Int. J. Mass Spectrom. Ion Proc. 108 R1-R10 